\title{
Plasma-Aided Manufacturing
}

\author{
J. Leon Shohet, Fellow, IEEE \\ (Invited Review Paper)
}

\begin{abstract}
Plasma-aided manufacturing is used for producing new materials with unusual and superior properties, for developing new chemical compounds and processes, for machining, and for altering and refining materials and surfaces. Plasmaaided manufacturing has direct applications to semiconductor fabrication, materials synthesis, welding, lighting, polymers, anticorrosion coatings, machine tools, metallurgy, electrical and electronics devices, hazardous waste removal, high-performance ceramics, and many other items in both the high-technology and the more traditional industries in the United States.
\end{abstract}

\section{InTERnATIONAL IMPACt OF Plasma-Aided MANUFACTURING}

$\mathbf{P}$ LASMA-Aided Manufacturing has a direct impact in the world economy in virtually every aspect of hightechnology industry as well as many of the more "traditional" industries of our nation. Industries in Japan and Western Europe have made major inroads into the plasma-aided manufacturing markets, which have drastically improved their competitive position. Our industrial colleagues tell us that if we can further develop the potential of plasma-aided manufacturing, it can make a significant contribution to the creation of markets worth billions of dollars and substantial increases in jobs.

Plasma-aided manufacturing encompasses a vast range of industrial applications, from thin-film sputter deposition, plasma polymerization, and microcircuit fabrication to welding, tool hardening, arc melting, synthesis of pure, ultrafine powders, plasma spraying, plasma sintering, and microwave generation [1]. It is also a topic that is inherently cross disciplinary and very much in need of university/industry collaborative research. It is also a topic that is ripe for major "break-throughs," which will be extremely important in preserving and increasing an industry's competitive position in the international marketplace.

The present and potential applications of plasma-aided manufacturing include:

- Fabrication of semiconductor integrated circuits and other electronic devices

- Hardening of tools, dies, and industrial metals

- Production of biocompatible and packaging materials for pharmaceuticals

- Anticorrosion and other coatings deposited on surfaces

Manuscript received February 1, 1991; revised May 6, 1991. This material is based upon work supported by the National Science Foundation through Grant ECD-8721545.

The author is with the Engineering Research Center for Plasma-Aided Manufacturing, University of Wisconsin, Madison, WI 53706.

IEEE Log Number 9102541.
- High-performance ceramics, including superconductors

- Production of new chemicals and materials

- Refining of metals

- Printing of polymer films

- Hazardous waste removal

- Welding

- Magnetic recording media

- Precise machining

- Lighting and displays

- Plasmas in electronic circuits and switchgear.

Estimates of the current potential markets for some of these areas are:

- $\$ 26$ billion in semiconductor processing

- $\$ 40$ billion in plasma electronics

- $\$ 2$ billion in tool and die hardening

- Many billions in thin polymer films for recording and medical uses.

Estimates of some new and exciting markets are:

- $\$ 50$ billion in metal corrosion protection

- $\$ 5$ billion in high-performance ceramics

- Many billions in markets for waste removal, metal refining, packaging, and pharmaceutical applications.

\section{Description of Industrial Plasmas AND PlaSma-Aided MANufaCturing}

Industrial plasmas are often only partially ionized, and therefore also contain neutral particles and/or free radicals, in addition to the charged particles. They can be divided into two categories: thermal and nonequilibrium (glow-discharge) plasmas. Their basic difference is that the former tend to have ion and electron temperatures roughly equal, and they tend to be at higher operating pressure than the nonequilibrium types. Both of these plasmas are of major importance. The central unifying factor between them is the fact that measurement and characterization as well as theoretical modeling of the plasma are needed for process control and optimization for high quality. In many cases, the techniques are similar.

Plasmas used in industry encompass a wide range of processes which can often be classified as a function of operating pressure. Fig. 1 shows a number of such manufacturing techniques. The vertical axis is the $\log$ of the operating pressure in torr, while the horizontal axis describes the general nature of the various processes. At low pressures, the collision frequency is low and the primary interactions are between charged particles and the material to be processed, so that the process is primarily physical in nature. As the pressure 


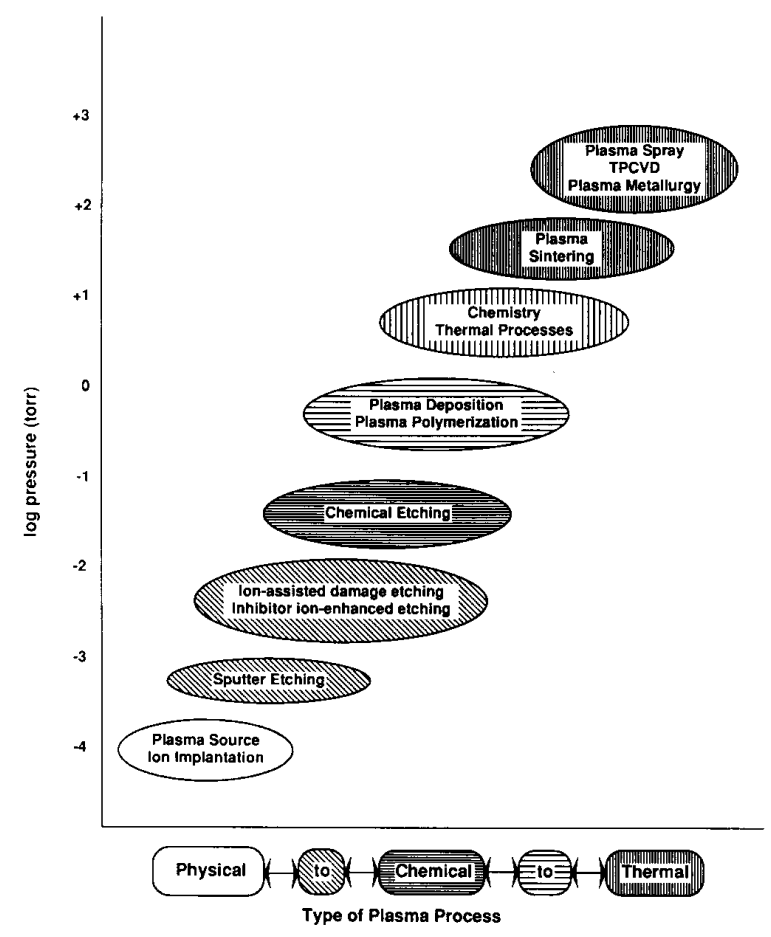

Fig. 1. Plasma processes arranged by operating pressure. As the pressure increases, the character of the process tends to move from physical to chemical to thermal in nature.

increases, collisions become more consequential and chemistry begins to play a more important role. As the pressure increases still more, and approaches $1 \mathrm{~atm}$, the plasma begins to act more like a thermal or heat source, substituting in many cases for combustion. At these pressures (thermal plasmas), ion and electron temperatures are roughly equal and are of higher density than the lower pressure (nonequilibrium plasmas) types where electrons tend to be hotter than ions.

Almost all applications of nonequilibrium plasmas simultaneously involve four processes: sputtering, etching, polymerization, and surface modification, even though a particular piece of equipment might be designed to use only one of them; e.g., an etching device [2]. Thermal plasma-processing applications are concerned with the interaction of high-temperature plasmas with gaseous reactants or particulate matter. The latter may be either injected into the plasma in the form of small particles (plasma spraying, plasma decomposition and synthesis, plasma reduction, etc.) or it may be exposed to the plasma in the form of bulk materials (melting and refining and other metallurgical applications) [3]. The goal of plasmaaided manufacturing is to control the generation and flux of ions, electrons, and free radicals and/or larger particles incident on a surface so as to modify that surface, either by depositing material on the surface (deposition) or removing material (etching or sputtering), or imbedding particles (ion implantation).

This process is very complex. The plasma is used to activate a chain of chemical reactions and to deliver activation energy to a surface (substrate). The plasma contains electrons, positive

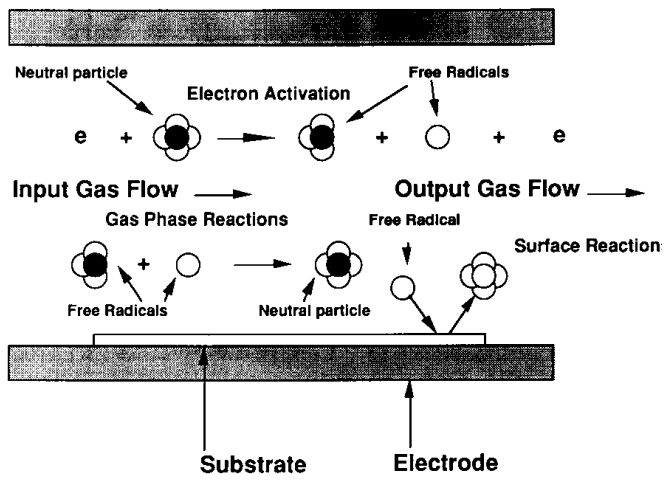

Fig. 2. Reactions taking place in a plasma-processing reactor.

and negative ions, neutral particles, and free radicals, all of which are often necessary for proper processing. Feedstock gases are flowed into the discharge where electron impacts dissociate and ionize the gas forming the free radicals and ions. These fragments further chemically react with the feedstock gases and other radicals as they diffuse and drift to the substrate. At the substrate, radicals and ions adsorb, bond, sputter, or otherwise react to modify the substrate while products are released back into the plasma. The combination of neutral species adsorption and positive ion bombardment can result in surface chemical reactions. If the products of the surface reaction are volatile, they leave the surface and etching results. If the products of the surface reaction are involatile, a surface film grows (deposition). If the particles remain on or just below the surface, implantation results. Fig. 2 shows a stylized view of a plasma-processing reactor with three types of processes occurring [4]. First, electron dissociation generates free radicals; second, the free radicals may recombine into other materials or back into the original components; and third, the free radicals and ions may travel to the surface, producing reactions there.

There are many, many operating parameters (external process variables) such as gas flow rates, gas composition, pressure, electrical power and frequency, substrate temperature, and reactor geometry. There are many, many process characteristics such as etch and/or deposition rates, spatial uniformity, selectivity, degree of anisotropy, and sensitivity. In the past, this complexity has resulted in process techniques being developed empirically rather than on the basis of fundamental principles. One of the reasons for this situation is that the external process variables listed above cannot simply regulate the internal process variables which actually govern the process. These variables are the energies, densities, and fluxes of electron and ions, neutral gas molecules, and free radicals produced in the reactor as a function of position and time. Determining the relationship between the internal process variables and the external process variables is the key to further advances in plasma-aided manufacturing.

As indicated above, plasma-aided manufacturing takes on a wide variety of apparently different forms in industry, but the techniques share many common characteristics and problems. Diagnostics, sensors, modeling techniques, and associated 
statistical methods developed for specific devices are usually applicable, with some modification, to many other devices. Problems addressed must have the direct attention of engineers and scientists who are experts in plasmas, chemistry, materials, statistics, microelectronics, and manufacturing, working as cross-disciplinary teams. The need for such teams is critical for the improvement of plasma-aided manufacturing, because no one person has yet been trained to be familiar with the details of all these fields.

\section{Industrial NeEdS AND PROBLEMS CONCERning Plasma-Aided Manufacturing}

Semiconductor electronics, which lies at the heart of virtually all modern manufacturing and high-technology products, is an area in which plasma-aided manufacturing has become essential. In the manufacturing of integrated circuits, for example, so-called "dry" processing which uses plasmas and/or particle beams for microfabrication is replacing "wet" chemical processing. Dry processing is required because of the ever-growing need for smaller and smaller separation between circuit elements. As critical device dimensions shrink to $1 \mu \mathrm{m}$ and below, wet processing becomes impractical for many required processing steps owing to problems of surface tension, linewidth control, undercutting of mask images, contamination, and the inability to achieve high aspect ratios. Dry processing techniques such as plasma etching and ion milling overcome these difficulties. In addition, the smaller device dimensions are dictating a move toward more complex and difficult metallizations such as refractory metals and their silicides. These metallizations are best deposited by plasma or plasma-assisted techniques such as sputtering or plasma-assisted chemical vapor deposition (PACVD). Current estimates of the size of the market in semiconductor plasma processing today approach $\$ 5$ billion per year, and it is predicted that this will increase annually.

The high-performance ceramics field is another area which is attracting increasing attention. Because plasma-produced ceramic powders are extremely fine and pure, they lend themselves to pressureless sintering, resulting in superior material properties. The present market for high-performance ceramics is about $\$ 5$ billion per year, with a projected U.S. annual growth of $28 \%$ for gas and humidity sensors, $25 \%$ for structural ceramics, $20 \%$ for cutting tools, and $10-14 \%$ for other applications. According to a report by the Long Term Credit Bank of Japan [7], high-performance ceramics will be among the three leaders (car electronics, office computers, high-temperature ceramics) in industry growth over the next 5 years. Japan has already begun to show results from these strong programs. A significant growth in automobile engine parts will begin in the 1990's. It is expected that the ultimate goal-the "all-ceramic engine"-will require minimal or no cooling and will give unprecedented gas mileage. Preliminary work in fabricating these materials, using plasma sintering, has already begun. The new field of high-temperature superconductors, which are, in fact ceramics themselves, can well be the beneficiary of plasma sintering in their manufacture.
Included in plasma-aided manufacturing are surface modification, plasma spraying, melting, thermal plasma synthesis, welding, and electrical discharge machining. In addition, plasma surface hardening, cleaning, nitriding, oxidizing, or other types of surface treatments preparatory to bonding or coating are major factors in today's technology. This is particularly true in high-technology areas where exotic or "difficult" materials such as ceramics, refractory metals, or composites must be processed.

The unique characteristics of plasmas (high effective temperatures, energetic particles, etc.) often offer the only practical means for such processing. Plasma spraying is already widely used for applying thick coatings of refractory materials and is one of the prime candidates for producing effective anticorrosion coatings, especially for high-temperature applications [8]. Losses due to metallic corrosion alone amount to more than $\$ 50$ billion per year in the U.S.

The plasma environment also offers opportunities for the synthesis of materials that would be difficult or impossible to produce in conventional chemical reactors, and for simultaneously synthesizing and depositing materials in a usable form, eliminating many subsequent processing steps. Plasma polymerization offers such an example. Under suitable conditions, monomers introduced into a plasma environment can polymerize [8], often producing polymers having characteristics that are not found in conventional polymerization. Plasma processing is very well-suited to producing polymers in the thin-film form which is required in many important applications such as coatings on other materials, multiple layers for magnetic recording tapes or disks, or "plastic" wrapping materials. Although it is not widely appreciated, the dollar volume for magnetic recording media is considerably larger than that for microcircuits. In the biomedical area, coatings of biocompatible polymers will permit continued progress in the development of sophisticated prosthetic devices, implantable replacement parts, and tools for medical monitoring and diagnostics. The recent developments in producing diamond and diamond-like coatings are often the result of a plasma-deposition process as well. The same type of processing can be used to decompose materials; for example, hazardous wastes [9]. This is presently done by passing the waste material-either in the form of a liquid spray or a fine powder-through an arc plasma.

Plasma electronics covers the range from plasma displays to arc-switching devices used in the power generation and transmission industry. In addition to the need for further development of these and related devices, there is a great need for improved materials for use in plasma environments. Deterioration of electrodes in arc devices, for example, as in power switchgear, is a constant and costly problem. The market for plasma electronics is also estimated to be upwards of $\$ 40$ billion per year.

Analytical instrumentation and systems development based on plasma technology has become increasingly important, and there is now a substantial market for mass spectrometers, ion accelerators and implanters, sputter-deposition systems, ion mills, plasma etchers, plasma torches, and thermal plasma reactors, as well as instrumentation for noninvasive diagnostics 


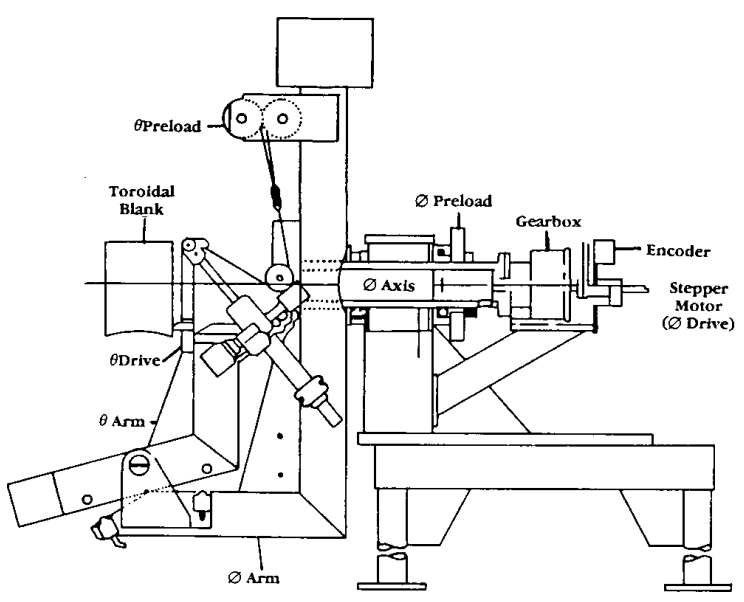

Fig. 3. Electrical discharge machining system for a magnet coil.

for plasma processes. Although much of this development has proceeded in an empirical, evolutionary manner, the manufacturers of instrumentation and plasma systems are now at the forefront of what is known about the generation, control, and behavior of plasmas and plasma/material interactions. Future progress in plasma-aided manufacturing will be greatly expedited if applications research is closely coupled with work on improved instrumentation, statistical methods, and manufacturing systems.

Plasmas are often involved in fabricating specialized mechanical parts. They are used for cutting and machining and often do so where no other process can be used. In spite of this, a representative of an industry which is heavily involved in electrical-discharge machining stated: "We still don't know enough about how the plasma actually cuts to be able to design the most competitive equipment." As an example of such machining, Fig. 3 shows a photograph of electrical discharge (plasma) machining of a magnet coil for a modular stellarator at the University of Wisconsin-Madison.

At an NSF workshop on Plasma Chemistry and Arc Technology in 1980 , one of the participants summarized the then present state of affairs as follows: ". . . plasma-surface reactions are either extremely important or dominate many processes where plasmas are used in manufacturing and research and development, and yet a basic understanding of these surface reactions is almost nonexistent." This situation still exists today. In order to put plasma-aided manufacturing on the firm scientific base that will be required for future progress, it will be necessary to foster genuinely collaborative work among plasma scientists and engineers, plasma chemists, materials scientists and engineers, statisticians, and industrial and manufacturing engineers from both universities and industry. Table I shows one view of the various types of complementary and cross-disciplinary activities that are needed.

Plasma-Aided Manufacturing is now at a critical point in its development. In the past, it has been possible to produce "industrial" plasmas for use in plasma-aided manufacturing with limited knowledge of the plasmas, their chemistry, their specific process control, quality and productivity improvement,
TABLE I

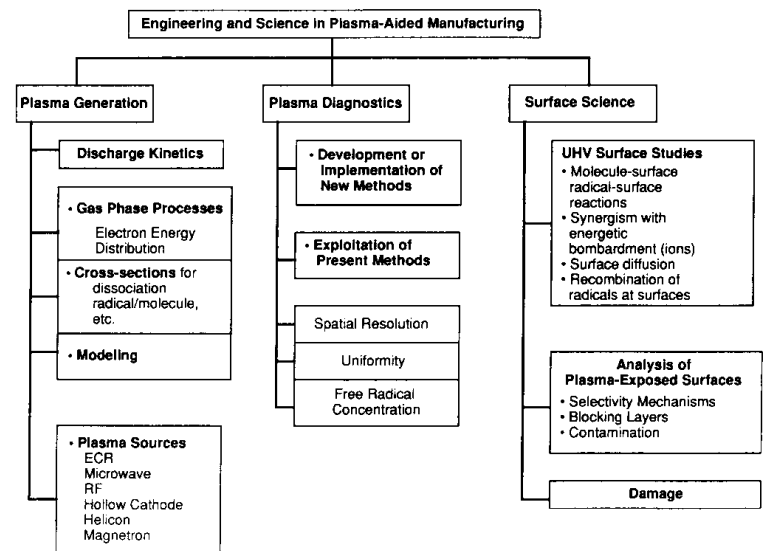

and sophisticated production techniques. Now our industrial colleagues tell us that, without an in-depth understanding of the variety of phenomena taking place and their application to the industrial environment, advances in this technology, and its efficient use, will occur at a diminishing rate. Specifically, our partners have expressed concerns about yields, end-point detection, reliability, and feasibility (both scientific and economic) of various plasma processes:

\begin{abstract}
"A large fraction of the down-time of our integrated-circuit assembly line is due to problems with the plasma system. We repair it electrically and mechanically, but it still doesn't operate properly as far as the plasma is concerned, and it takes us an incredible amount of time to adjust it to work again. Yet, without plasma processing, we are dead in the water."
\end{abstract}

This is the opinion of the manager of a semiconductor electronics fabrication facility whose responsibility is the plasma "tools" used in their manufacturing process [12].

In February 1985 the National Research Council released a report of the National Materials Advisory Board on Plasma Processing of Materials [13]. The report states that "Efforts should be made to focus basic research on thermal and nonequilibrium plasmas at universities, with a view to establishing in the long term a few Centers of Excellence covering all relevant aspects of the subject." The National Science Foundation's Engineering Research Center for Plasma-Aided Manufacturing at the University of Wisconsin-Madison is such a center.

\section{The Plasma Processes}

Nearly every problem in plasma-aided manufacturing requires a cross-disciplinary approach to develop and improve the manufacturing techniques and to ask and resolve the basic questions. In general, plasma-aided manufacturing is one or more steps in a manufacturing process, but not usually the entire process. Thus, all of these specific techniques must ultimately be integrated into systems in order that they can be 
utilized in industry. As a result, plasma-aided manufacturing involves not only the development of new techniques and processes for manufacturing products, but their integration into a complete manufacturing system to produce these products.

In particular, many of the problems encountered in the semiconductor manufacturing industry today involve reliability, uniformity, and speed of production of plasma systems. A recent conversation with a representative of a major semiconductor manufacturing company revealed that plasma equipment purchased "off-the-shelf" was actually not fully developed. As a result, the company was required to work with the vendor, essentially completing the development work in the field for periods of time upwards of 1 year before the equipment was in satisfactory working condition. Following is a description of four areas of plasma-aided manufacturing, together with some proposed directions for addressing the problems associated with them.

\section{A. Plasma Modification of Materials}

This process is designed to improve the surface properties of materials without changing its bulk properties by implantation of specific ions into the material. It is becoming economically attractive in Japan, Europe, and the U.S., and can be done on metals and alloys as well as semiconductors, ceramics, insulators, and polymers. Some of the surface properties that can be modified by this process are: hardness, adhesion, corrosion, superconductivity, fatigue, friction, oxidation, resistivity, toughness, catalysis, wear, and dielectric properties.

A novel technique for ion implantation with a plasma source that offers considerable advantages relative to existing techniques has been developed. In conventional ion implantation devices, an ion beam is extracted from a plasma source, accelerated to the desired energy, and then transported to the target. Typical beam currents are very small (in the microampere range) and the beam "footprint" area is less than $1 \mathrm{~cm}^{2}$. In order to process large-scale targets and in order to avoid shadowing if the target is nonplanar, a combination of beam rastering and target rotation during the implementation is required.

In the plasma-source ion-implantation process, a dc discharge, the strongest electric field is in the cathode sheath, which accelerates positive ions to the cathode. If the pressure is kept low enough so that an arc discharge does not occur, positive ions can be accelerated to strike the cathode with energies of $100 \mathrm{keV}$ or more. With these energies, ions can implant themselves in the cathode surface, which has important applications in improving tool hardness and lubricity. At lower energies, implantation can benefit the microelectronics industry. Fig. 4 is a schematic drawing of a plasma ionimplantation system showing the vacuum chamber in which the high potential required for plasma implantation is applied between the walls of the chamber and the material to be implanted.

In the plasma source ion-implantation process, a pulsed $\mathrm{dc}$ discharge, the strongest electric field is in the cathode sheath, which accelerates positive ions to the cathode. If the pressure is kept low enough so that an arc discharge does not occur, positive ions can be accelerated to strike the cathode with energies of $100 \mathrm{keV}$ or more. With these energies, ions can implant themselves in the cathode surface, which has important applications in improving the hardness and lubricity of tools and other products which are subjected to mechanical wear. At lower energies, implantation can benefit the microelectronics industry. Fig. 4 is a schematic drawing of a plasma ionimplantation system showing the vacuum chamber in which the high potential required for plasma implantation is applied between the walls of the chamber and the material to be implanted.

The implantation process begins with the application of a pulsed, high-voltage, negative dc potential between the material to be implanted and the vacuum chamber wall. As the potential becomes more and more negative, the sheath moves into the plasma itself. As the sheath moves through the plasma, electrons are "reflected" from the plasma-sheath boundary and kept in the plasma. It should be pointed out that the plasma-sheath boundary does not move the ions as it travels into the plasma. Rather, since the ions are more massive, the boundary sweeps through the ions. When the ions find themselves on the other side of the boundary, they are affected by the electric field in the sheath, which tends to accelerate them to the cathode.

The sheath tends to be in the Child-Langmuir limit. In one dimension, we may write an equation for the ion current that appears as the sheath moves into the plasma as the following:

$$
n q \frac{d s}{d t}=J_{\text {ion }}
$$

where $s$ is the position of the sheath at time $t, n$ is the ion density, and $q$ is the ion charge. For the Child-Langmuir conditions to be satisfied, then:

$$
J_{\text {ion }}=n q \frac{d s}{d t}=\frac{4 \varepsilon_{0}}{9} \sqrt{\frac{2 q}{m_{i}}} \frac{V^{3 / 2}}{s^{2}} .
$$

This can be integrated directly to obtain $s(t)$ and the sheath velocity provided that $V(t)$, the voltage applied to the cathode, is known. The total number of ions incident on the cathode can then be obtained by integrating the above equation with respect to time. This gives the "dose" of ions implanted on the cathode. The dose of ions and their energy determines the depth at which the ions have been implanted and the properties of the modified material.

Currently, identification of the factors and their interactions which influence this process are poorly understood from the standpoint of the behavior of the material which is implanted. To further advance this field, an understanding of how the bombarding particles interact with the base material and how to apply this knowledge to manufacturing techniques will be an opportunity for industry to further exploit this technology. Two aspects of this technique: (i) surface hardening and wear resistance, and (ii) corrosion and oxidation resistance are particularly important. Statistical methods of design of experiments and, in particular, response-surface methods, are ideally suited to explore the interaction effects and are a crucial tool for further development in this area. 


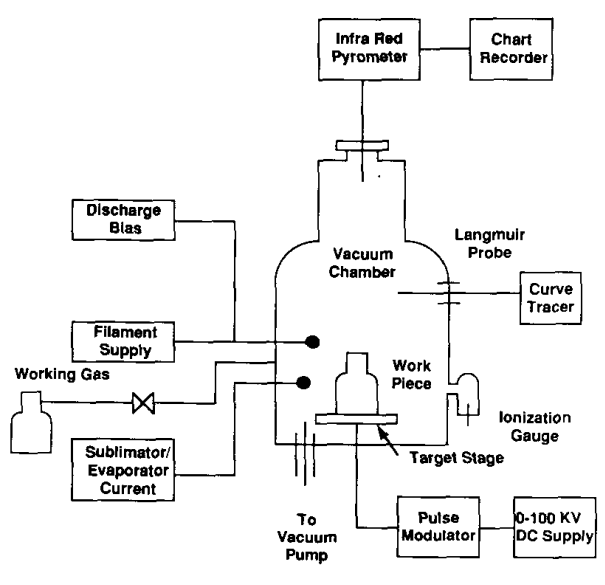

Fig. 4. PSII System.

It has been conclusively demonstrated that ion implantation can beneficially modify the surface-sensitive mechanical properties of steels. Fatigue life has been extended by as much as a factor of 2 , the coefficient of sliding resistance reduced by as much as a factor of 100 , and the wear resistance increased by a significant amount. Most studies have concentrated on the use of nitrogen ions, but the use of $\mathrm{C}, \mathrm{B}, \mathrm{Ti}$, and Mo ions have also shown promising results.

Recent work in the College of Engineering of the University of Wisconsin-Madison [14] has shown remarkable improvement in properties of: (a) a nitrogen-implanted alloy die steel, and (b) nitrogen-implanted aluminum tools for machining high-temperature alloys. These projects are continuing in collaboration with several companies, and the effects of implanting nitrogen into a surface previously enriched-i.e., by vapor deposition-with carbon and/or boron is now being examined. Ultimately, this work will be extended to cover the effect of incident nitrogen ion energy (depth of hardening), concomitant fatigue properties, characterization of the microstructure by TEM/AEM, and mechanical properties versus depth by nano-hardness measurements. These studies are a prerequisite in controlling the manufacturing process and require statistical experimental design techniques for their implementation.

\section{B. Plasma Etching and Microwave Processing for Microelectronics}

Pattern transfer to a wafer by plasma etching [5] has become an indispensable technique for fabricating semiconductor devices whose critical feature dimensions are less than $3 \mu \mathrm{m}$. Etching can be done with wet chemicals, such as acids. With plasma etching it is possible to etch faster in the direction perpendicular to the surface of the material than parallel to the surface, resulting in anisotropic etching. This allows finer features to be etched, allowing many more transistors and diodes to be placed on a microchip. Anisotropic etching is the key to making smaller, faster, and cheaper chips. Plasma etching is a purely chemical process involving neutral atoms and/or free radicals which react with the surface. Reactive-ion etching (RIE) is defined as plasma etching with required simultaneous energetic ion bombardment of the processed surface. In fact, the most important reason that RIE has replaced wet chemical etching in microfabrication technology is the ability of RIE to accomplish anisotropic etching. The following materials used in microfabrication readily lend themselves to plasma etching in that they form volatile compounds under proper conditions:

$\begin{array}{ll}\text { silicon } & \text { silicon dioxide } \\ \text { silicon nitride } & \text { photresist } \\ \text { polyimide } & \text { aluminum } \\ \text { tungsten } & \text { titanium } \\ \text { gallium arsenide } & \text { indium phosphide. }\end{array}$

The following materials do not readily form volatile compounds, at least at or near room temperature:

nickel
iron
copper
metals from groups I or II in the Periodic Table.

In addition to the applications in microfabrication technology, the ability of RIE processing to eliminate the large quantities of liquid chemicals needed for wet processing is a decided advantage.

With energetic ion bombardment, a synergistic relationship often exists and etching rates can be faster than with either plasma or ions alone [6]. Some reactions are unaffected or inhibited by ion bombardment, such as $\mathrm{Al}-\mathrm{Cl}$ and $\mathrm{Cu}-\mathrm{Cl}$. Here, plasma polymerization can play a role. In halocarbon discharges with a deficiency in the halogen often brought upon by etching, polymer films will be deposited on surfaces in contact with the discharge, and their etch rates are strongly decreased. Polymers can form in etching plasmas when saturated halocarbon gases such as $\mathrm{CF}_{4}$ are used. Some of the fluorine is used in the etching process, leaving $\mathrm{CF}_{2}$, for example. This is then able to form polymers such as polyethylene. However, surfaces subjected to ion bombardment will be relatively free of polymer (i.e., bottom surfaces), whereas surfaces not being bombarded by energetic ions (sidewalls) will be coated with a thin polymer film. This allows etching to proceed on the bottom, but the polymer passivates (inhibits) the etching process on the sidewalls and results in a directional etching process which can produce submicron patterns. This is shown in Fig. 5. The sidewall film can be removed, often by wet chemical etching after processing.

Table II shows some typical characteristics of RF plasmas used for reactive ion etching in the United States.

In the actual practice of plasma etching, it is required to monitor and control a wide variety of processing variables involving three major decision areas: 1) the electrode configurations for the plasma and the current, voltage, and frequency applied; 2) the reactant gas composition flow rate and pressure; and 3) the nature and kinetics of the surface reactions of the plasma-generated species. This inherent process flexibility provides a need for engineering statistics and manufacturing technology for operation and control. The emphasis here is in the identification of reactive species in the plasma and the characterization of the surface reactions which occur. 
TABLE II

Typical Characteristics of RF Plasmas Used for Reactive Ion

\begin{tabular}{ll}
\multicolumn{1}{c}{ QTCHING IN THE UNITED STATES } \\
\hline \multicolumn{1}{c}{ Quantity } & \multicolumn{1}{c}{ Typical Values } \\
\hline RF Power Density & $0.05-1.0 \mathrm{~W} / \mathrm{cm}^{2}$ \\
RF Frequency & $10 \mathrm{kHz}-27 \mathrm{MHz}$ \\
Pressure & $0.01-0.2 \mathrm{torr}$ \\
Gas Flow & $10-200 \mathrm{sccm}$ \\
Water Temperature & $-120^{\circ}-300^{\circ} \mathrm{C}$ \\
Gas Temperature & $300-600 \mathrm{~K}$ \\
Electron Temperature & $3-30 \mathrm{eV}(\mathrm{bulk}$ of plasma) \\
Ion Temperature & $0.5 \mathrm{eV}(\mathrm{bulk}$ of plasma) \\
& $10-500 \mathrm{eV}$ after traversing sheath \\
Gas Density & $3.5 \times 10^{14}-7 \times 10^{15} \mathrm{~cm}^{-3}$ \\
Ion Density & $10^{9}-10^{10} \mathrm{~cm}^{-3}$ \\
Electron Density & $10^{9}-10^{10} \mathrm{~cm}^{-3}$ \\
Ion Flux & $10^{14}-10^{15} \mathrm{~cm}^{-2} \mathrm{~s}^{-1}$ \\
Free Radical Flux & $10^{16} \mathrm{~cm}^{-2} \mathrm{~s}^{-1}$ \\
Neutral Flux & $3.6 \times 10^{18}-7.2 \times 10^{19} \mathrm{~cm}^{-2} \mathrm{~s}^{-1}$ \\
\hline
\end{tabular}

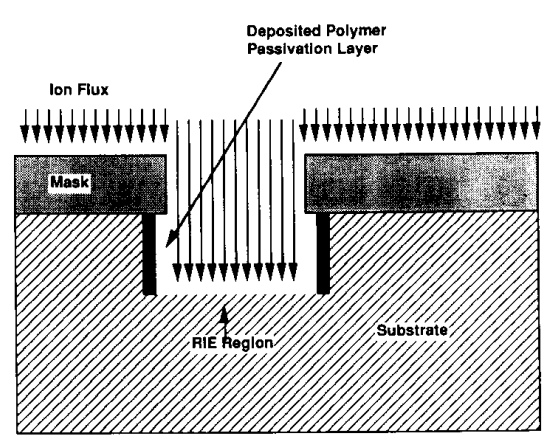

Fig. 5. Anisotropic etching.

The basic needs to improve plasma-etching techniques are: 1) to characterize in stages the gas-phase species present in a plasma using conditions appropriate for reactive ion etching; 2) to study the surface reactions which occur both on the photoresist covered and on the uncoated semiconductor surfaces; 3 ) to devise analytical diagnostic schemes to monitor and control a plasma-etching process leading to submicron minimum linewidth devices; 4) to utilize plasma modeling expertise to develop better understanding and control over the plasma-etching process; and 5) to increase yield, improve uniformity, and quality by using statistical methods of design and analysis for process optimization. These objectives can be accomplished by bringing a number of analytical techniques to bear on the plasma systems and by examining the etchedimage profiles. However, only with sufficient diagnostics and sensors can one hope to determine the importance of the internal process variables, and that statistical techniques of experimental design and analysis can be used to reveal direct relations between the variables.

\section{Plasma Deposition and Polymerization}

A variety of plasma processes are currently used to deposit materials on surfaces. Several of them are described below. They are based on the fact that surrounding an electrode, a plasma sheath often develops. Such a sheath can have a considerable potential drop across it, which can be used to accelerate positive ions to the electrode. The impact of the

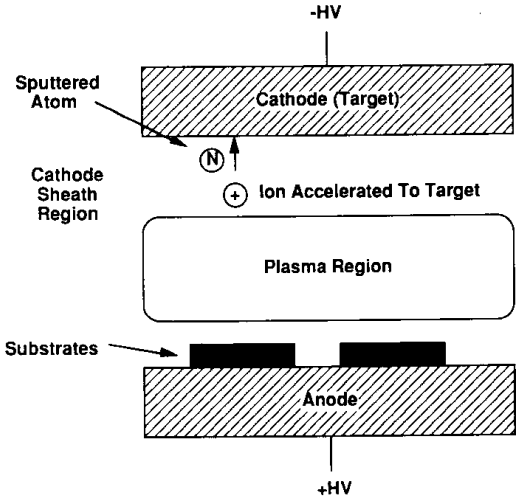

Fig. 6. Plasma sputtering system.

ions on the electrode can remove material from the electrode or serve as an "activator" for free radicals that travel to the electrode. A drawing of a dc planar electrode plasma reactor exhibiting the plasma and sheath regions is shown in Fig. 6.

1) Sputtering: Ions, when striking the cathode, can produce a "collisional cascade," which may result in atoms being ejected from the cathode surface-sputtering. Sputtering "yields," the number of atoms per incident ion, are functions of the input energy and the ion mass, as well as the target composition. The sputtered atoms can then deposit on the surface of a substrate material, producing a "thin film."

The applied voltage which is required to drive the current through the system is a function of the system pressure. The rate of thin film formation on the substrate will depend on the amount of sputtering at the target; this in turn depends on the ion flux on the target and on the ion energy and hence on the voltage applied to the system, which then determines the sheath voltage at the target. The choice of the sputtering pressure and the implied choice of the $V-I$ characteristic are thus important.

The actual sputtering gas is often not critical, but in many cases it is required that the gas does not chemically react with the target, film, or substrate, so often inert gases are used for sputtering. In addition, since sputtering gases may also become entrapped in the growing film, use of inert gases will not cause chemical reactions with the growing film.

The choice of pressure for sputtering is based on the need to have a high deposition rate balanced by the requirement to keep the discharge going. Below about 30 mtorr, the discharge current and the sputtering rate in the discharge are quite small, because the rate of ionization decreases with pressure and thus the discharge current for a given voltage will decrease with pressure.

2) Ion Plating: This is a process which takes place in a reactor similar to that used for sputtering, and is often used as a precursor or concurrent process with sputtering. It is used to provide good adhesion between the film and a surface and/or to modify the substrate surface before deposition of a film. It provides high-energy flux to the substrate surface, giving a high surface temperature, thus enhancing diffusion 
and chemical reactions without bulk heating. Ion plating can also physically mix the film and substrate material.

3) Sputter Etching: Sputter etching is the process which occurs when material is removed from a surface by sputter ejection. Since sputtering results from bombardment by ions which move along electric field lines and because field lines are always perpendicular to an equipotential surface, then etch profiles are inherently vertical (anisotropic), in contrast to the isotropic profiles observed with wet chemical etching. Sputter etching can be carried out in a conventional sputtering system with an in situ glow discharge or with an externally generated ion beam. When using either a plasma or an ion source, there is the possibility of charge-exchange collisions occurring with neutral atoms in the gas-producing energetic neutrals which are not affected by the electric field lines and therefore do not follow them directly to the target. In addition, sputtered material from the target, mask, or mask support could suffer collisions with the gas and be scattered back onto the substrate. This could lead to poorer results.

4) Plasma-Assisted Chemical Vapor Deposition (PACVD): PACVD consists of the technique of forming solid deposits by initiating chemical reactions in a gas with an electric discharge. Its chief advantage over thermal CVD is the ability to deposit films at relatively low substrate temperatures (typically less than $300^{\circ} \mathrm{C}$ ). Such films do not severely stress the substrates. PACVD is especially useful for materials which might either vaporize or melt, flow, diffuse, or undergo a chemical reaction at the higher temperatures. The major limitation of PACVD is that deposition of pure materials is virtually impossible. This can be either an advantage or a disadvantage, depending upon the application.

5) Plasma Polymerization: Plasma polymerization is very similar to PACVD. In many cases the difference is that the latter is concerned with the deposition of inorganic materials, and in the former, the deposition of organic materials which are often polymeric in nature. As an example, if an unsaturated fluorocarbon gas such as tetrafluroethylene is injected in a plasma, a Teflon-like film can be deposited on a substrate. The deposited polymer is often highly cross-linked and many high-quality films are formed which cannot be deposited by other means.

A polymer is normally built from a monomer precursor, but it can also be used to refer to a characteristic bonding structure of amorphous films. A polymer-like film differs from a glass by having less cross bonding of the major chemical components of the film, and instead, having a great deal of hydrogen or halogens saturating the dangling bonds. Thus polymerization is often used to refer to the formation of polymer-like amorphous films even if no monomer exists. Some plasma polymers have been formed that appear to consist of linked monomers of the starting gas. Most commonly, however, the plasma decomposes the starting gas into fragments. These fragments either nucleate into a polymer film at the surface, or polymerize themselves into chains and clusters. The relative magnitude of gas or surface polymerization is sensitive to the power, pressure, and choice of starting material. Polymerization reactors are often quite different in construction from parallel-plate reactors used for deposition and etching.

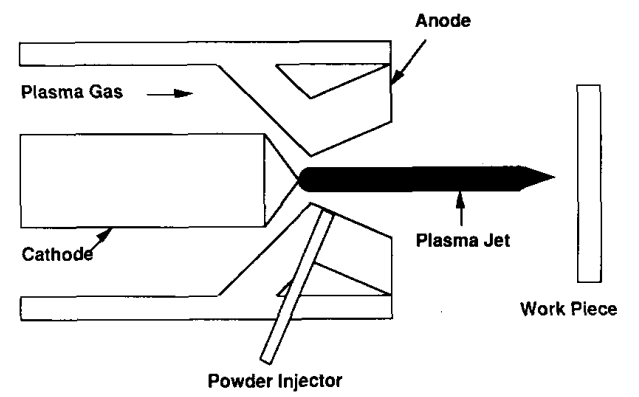

Fig. 7. A plasma torch.

\section{Thermal Plasma Spraying, Synthesis, and Sintering}

1) Plasma Spray: This process is of interest because of its ability to provide surface protection. Turbine blades of aircraft engines, for example, are protected by anticorrosion (super-alloys) and by thermal-barrier coatings (ceramics) applied primarily by low-pressure plasma deposition (LPPD). In addition, LPPD is considered to be a viable manufacturing technology for producing monolithic, nearnet shapes of metallic as well as ceramic materials. Rapid solidification, which is inherent to the plasma-spray process, may also produce desirable properties of coatings or monolithic bodies, because the process results in fine-grain structures and, in extreme cases (certain metallic alloys), in amorphous structures which reveal unusual properties in terms of strength and corrosion resistance.

Powder is heated to near or above its melting point in a plasma torch and is accelerated by a plasma gas stream toward a substrate, as shown in Fig. 7. On impact, the powder forms a coating consisting of many layers of overlapping thin lenticular particles--"splats." Almost any material that can be melted without decomposing can be used to form the coating. As with most plasma processes, the variables include: type of gases, flow rates, anode design, power level, and the location in the plasma torch where the powder is injected.

Most powder used for plasma spray is between 5 and 60 $\mu \mathrm{m}$ in size. To achieve uniform heating and acceleration of a single component powder, as narrow a size distribution as possible is preferred. Fine powders are accelerated and heated more rapidly, but tend to lose momentum more rapidly when spraying at longer distances from the torch. This generally results in denser, but more highly stressed, coatings. Virtually all plasma coatings require a roughened substrate surface, often done with grit blasting.

In spite of considerable advances of the plasma-spray technology over the past 20 years, the plasma-spray process as such is still poorly understood. In particular, there are no welldefined correlations available between the plasma parameters of the spray torch and the properties and quality of the coatings produced.

Experimental and analytical studies should address the three generic problem areas of plasma spraying: the torch parameters, the injection of particles, and the evaluation of the coating. As a first step, modeling work in parallel with relevant experiments will establish the plasma conditions in the jet 
(temperature, and velocity fields, electron concentrations) as a function of the plasma torch parameters. Besides spectrometric, Thomson scattering, and laser-Doppler measurements in the plasma jets, enthalpy probes will be applied for measuring local enthalpies, velocities, and gas entrainment into the jet, especially in the downstream sections of the highly turbulent plasma flame. Existing models for turbulent jets in cold flow have to be modified to account for strong variations of thermodynamic and transport properties across the jet.

2) Plasma Synthesis: Synthesis of materials, especially of refractory materials in thermal plasmas, is an emerging technology with the potential of providing ultrafine and ultrapure powders suitable for pressureless sintering. Development of this manufacturing technology has been severely hampered by the lack of an engineering base which is necessary for developments on an industrial scale. Work needs to be done to establish such a base.

Emphasis will be on the development, analysis, and diagnostics of relatively simple reactors, suitable for the synthesis of refractory fine powders of carbides, nitrides, and some metal oxides. Diagnostic methods will include emission spectroscopy, laser-Doppler anemometry, laser-induced fluorescence spectroscopy, and high-speed photography. The refractory powders will be analyzed using primarily electron microscopy, X-ray diffraction, BET, and Auger spectroscopy. Modeling work will include arc plasma, plasma jet, and RF plasma reactors. Another part of the modeling work will be concerned with particle injection, particle motion, heating, melting, and evaporation in the plasma, including the ensuing chemical reactions, quenching, and product formation.

3) Plasma Sintering: Plasma sintering is a new technology for the rapid sintering of ceramic materials, which are attracting increasing attention for a wide spectrum of uses, including structural, electronic, and biological applications. In the U.S., this technology has been pioneered by L. Johnson at Northwestern University, and results of laboratory-scale investigations have also been reported from Japan. Thermal plasmas represent extremely strong and easily controllable heat sources for sintering, reducing the required processing time by orders of magnitude compared to conventional technologies. At the same time, rapid sintering may result in unique and desirable structures and properties of the sintered materials. For example, the auto industry and other manufacturers of internal-combustion engines expect that sintered materials will revolutionize engine technology, because engines made of these sintered materials will not need cooling like today's metallic engines.

A general requirement for developing plasma sintering into a viable technology is a thorough understanding of plasma heat transfer during the sintering process. A model for describing the heating transfer process is required. A single, simple model will not be able to describe this process over the entire range of potential operating pressures (10 atmospheres to 1-2 atm), because the conditions encountered in the boundary layer will vary from continuum flow to molecular flow. At low pressures, for example, electric charges accumulating at the surface of the sample to be sintered will have a strong influence on the heat transfer process. In contrast, at high pressures, this effect will be substantially reduced or even negligible, compared to other heat-transfer mechanisms.

\section{SUMmary}

For successful application of a plasma-aided manufacturing process, two requirements must be met. First, the process must be developed. For a new plasma process, much time and money can be saved if the understanding of how the plasma, chemistry, and surface reactions combine to produce a specific result is known from experience and/or predictive modeling. Second, the economic benefits of the process must be well understood before the process can be utilized. If the process is the only way in which a material can be fabricated, the economic issues are not quite as important. However, if there is a competing technology, economics will be the most important factor in determining the acceptance of a process by industry.

\section{REFERENCES}

[1] J. L. Shohet, "Plasma science and engineering," in Encyclopedia of Science and Technology. San Diego: Harcourt, Brace, Jovanovich, 1987.

[2] D. M. Manos and D. L. Flamm, in Plasma Etching, D. M. Manos, Ed. San Diego: Harcourt, Brace, Jovanovich, 1989.

[3] Special Issue on Thermal Plasma Processing, Plasma Chem. Plasma Process., vol. 9 (suppl.), 1989.

[4] L. E. Kline and M. J. Kushner, "Computer simulations of materials processing discharges," Crit. Reviews in Solid State and Materials Sci., vol. 16, no. 1, pp. 1-36, 1989.

[5] Plasma Process. Symp. Proc., vol. 68, J. W. Coburn, R. A. Gottscho, and D. W. Hess, Eds. Pittsburgh, PA: Materials Res. Soc., 1986.

[6] J. W. Coburn, Plasma Chem. Plasma Process., vol. 2, no. 1, 1982.

[7] H. Kimura, Fine Ceramic Industry in Japan: Current Developments (LTCB Res. Special Issue, Japan's High Technol. Industry, vol. 3). Tokyo: Long Term Credit Bank of Japan, 1984

[8] H. Herman, Sci. Amer., vol. 259, p. 112, 1988.

[9] H. Yasuda, Plasma Polymerization. New York: Academic, 1985

[10] J. V. R. Heberlein, W. J. Melilli, S. V. Dighe, and W. H. Reed, in Proc. Int. Symp. on Plasma Chem. (Pugnochiuso, Italy), 1989, p. 10

11] G. E. P. Box, W. G. Hunter, and J. S. Hunter, Statistics for Experimenters: An Introduction to Design, Data Analysis, and Model Building. New York: Wiley, 1978.

[12] L. Ephrath, private communication.

[13] Plasma Processing of Materials. Washington, DC: Nat. Acad. Sci., 1985.

[14] J. R. Conrad, J. L. Radtke, R. A. Dodd, F. J. Worzala, and N. C. Tran, J. Appl. Phys., vol. 62, p. 4591, 1987.

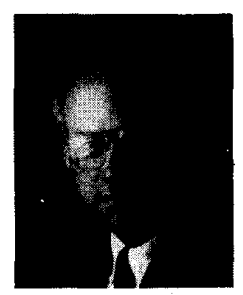

J. Leon Shohet (S'56-M'62-SM'72-F'78) received the Ph.D. degree in electrical engineering from Carnegie-Mellon University in 1961.

He served on the faculty of The Johns Hopkins University before joining the University of Wisconsin faculty in 1966, and was appointed Professor of Electrical and Computer Engineering in 1971. He currently serves as the Director of the University's NSF Engineering Research Center for PlasmaAided Manufacturing and the Torsatron/Stellarator Laboratory. $\mathrm{He}$ is the author of a textbook on plasma science and over 90 journal articles and more than 300 conference papers. He holds five patents. His research interests are in plasma-aided manufacturing; fusion, especially waves, instabilities, heating, confinement, and diagnostics; communications; magnetohydrodynamics; electromagnetic field theory; biophysics; quantum electronics; and lasers.

Dr. Shohet is a Fellow of the American Physical Society. He received the Frederick Emmons Terman Award of the American Society of Engineering Education, the Merit Award of the IEEE's Nuclear and Plasma Sciences Society, the IEEE Centennial Medal, and the IEEE Plasma Science Prize. 